\title{
Modeling Decontamination by Downstream Plasma Cleaning using Both Quartz Crystal Microbalance and UV-Visible Spectroscopic Data
}

\author{
Christopher G. Morgan and Ronald Vane \\ XEI Scientific, Inc., 1755 E. Bayshore Rd., Suite 17, Redwood City, CA 94063
}

Carbon contamination in scanning electron microscope (SEM) chambers can result in poorer image quality. Downstream plasma cleaning is an effective means for removing carbon contamination from SEM chambers. The plasma device is mounted on an unoccupied port on the SEM. When in use, the device creates oxygen radicals using a small leak of oxygen containing gas and a low power $(5-20 \mathrm{~W})$ radio frequency $(\mathrm{RF})$ plasma. The oxygen radicals then flow through the SEM chamber, ashing hydrocarbons. The rate of carbon decontamination has been shown to be dependent on a number of factors: RF power level, pressure of chamber during cleaning, distance of contamination from oxygen radical source, geometry of chamber, and type of oxygen containing gas used [1]. Decontamination is also dependent on the speed of the pumping system used to evacuate the SEM chamber.

The primary objective of this research is the development of a quantitative downstream plasma cleaning model so that cleaning rates can be estimated for any vacuum system. By creating chemical models of both the oxygen radical creation within the plasma and oxygen radical reactions once they leave the plasma, estimates of cleaning rates can be obtained. These models will be based on physical data from two kinds of experiments varying the parameters listed in the above paragraph.

The first experiment uses a quartz crystal microbalance (QCM) to measure a cleaning rate in a given area of a vacuum chamber; details this method has been reported by this group [2]. A simple model of the decontamination process on the QCM has been developed [3]. Using pure oxygen gas or dry room air, the model assumes only oxygen radicals contribute to decontamination, while nitric oxide (NO) inhibits the removal of the contamination. Six gas-surface reactions are assumed to occur in the model. A sample of a model fit to the data is shown in Figure 1. Results for industrial oxygen are shown in Figure 2. By locating the QCM holder in different locations of a vacuum chamber, a map of oxygen radical concentrations as a function of distance from the plasma can be made. This map can then be compared to a steady-state box model of oxygen radical concentrations in the chamber by using a standard database of gas phase reaction rates [4].

The second experiment uses UV-visible spectroscopy to determine the species found within the plasma itself. In plasma created with room air or dry air, the main emissions seen are from $\mathrm{N}_{2}$ and NO. Preliminary spectroscopic results indicate the presence of hydroxyl radical when room air is used and have confirmed that oxygen radical is present in the plasma. These studies show that the nitrogen ion number density increases with decreasing chamber pressure as seen in Figure 3.

Nitrogen ions remove oxygen radicals from the plasma and decrease cleaning efficiency. The three body recombination removal rates for oxygen radicals also decrease with lower pressure, and the net result is the observed increase in cleaning efficiency with lower pressure. The spectroscopic data 
suggests that the excitation, dissociation and ionization of $\mathrm{N}_{2}$ and $\mathrm{O}_{2}$ gases in the plasma occur in stepwise processes. A second steady-state model of the plasma, assuming stepwise excitation of $\mathrm{N}_{2}$ and $\mathrm{O}_{2}$ gases, will be compared to the spectroscopic data

\section{References}

[1] C. G. Morgan, M. M. Gleason, and R. Vane, Microscopy Today 15 (5) (2007) 22; C. G. Morgan, M. M. Gleason and R. Vane, Microsc. Microanal. 13 (Suppl 2) (2007) 1736

[2] M. M. Gleason, C. G. Morgan and Ron Vane, Microsc. Microanal. 13 (Suppl 2) (2007) 1734

[3] C. G. Morgan and R. Vane, Micros. Microanal. 14 (Suppl 2) (2008) 1280

[4] W. B. DeMore, S. P. Sander, D. M. Golden, et al., Chemical Kinetics and Photochemical Data for Use in Stratospheric Modeling, Eval. No. 12, JPL Publication 97-4, 1997; G. Brasseur and S. Solomon, Aeronomy of the Middle Atmosphere, $2^{\text {nd }}$ Ed., D Reidel Publishing Company, 1986

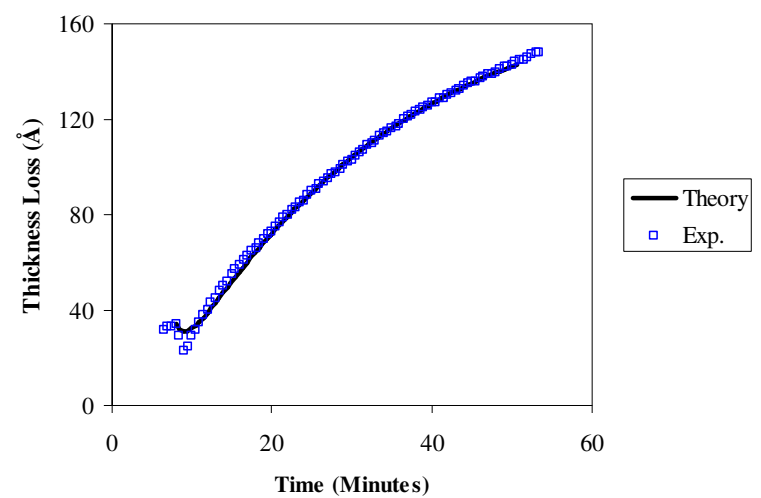

Figure 1: Comparison of model fit to quartz crystal microbalance data. Industrial oxygen gas used, chamber pressure is 0.5 Torr, forward RF power is $10 \mathrm{~W}, 141 \AA$ pump oil contamination. The QCM holder is $15 \mathrm{~cm}$ from plasma source.

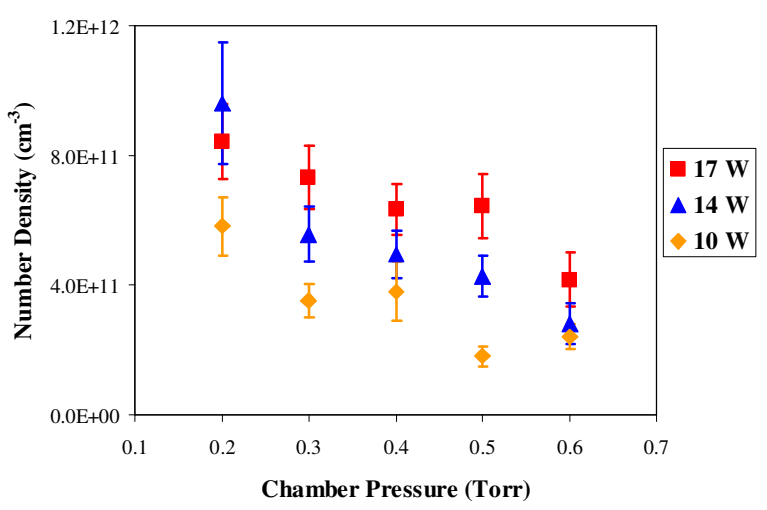

Figure 2: Model derived oxygen radical number density $15 \mathrm{~cm}$ from plasma source. Initial contamination is $\sim 10 \mathrm{~nm}$ pump oil, and industrial oxygen gas used. Number density of $\mathrm{O}$ for three different forward RF powers derived (10 W, $14 \mathrm{~W}$, and $17 \mathrm{~W})$. Error bars reflect difference between model fit and data.

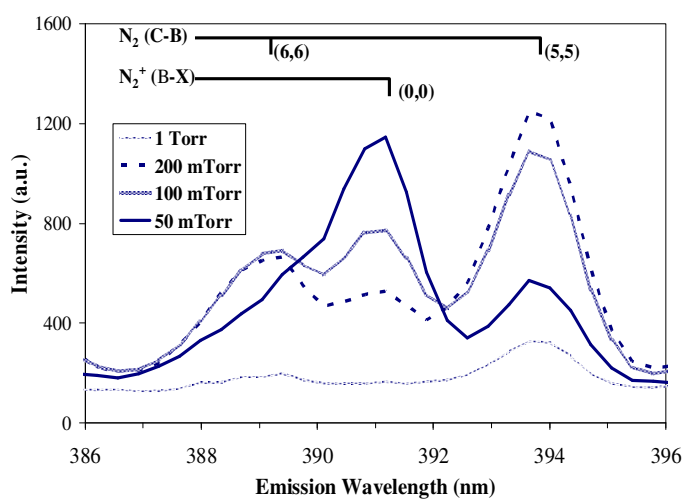

Figure 3: Photoemission spectra in the $386-396 \mathrm{~nm}$ region of room air plasma created with $14 \mathrm{~W}$ forward RF power. Each trace is taken under different chamber pressure. Emission due to $\mathrm{N}_{2}{ }^{+}$is the center peak around $391 \mathrm{~nm}$; emissions due to $\mathrm{N}_{2}$ are the side peaks around 389 and $394 \mathrm{~nm}$. 